\title{
Describing Inorganic Nanoparticles in the Context of Surface Reactivity and Catalysis
}

\author{
S. Carenco ${ }^{a}+$ \\ Fabrication of inorganic nanoparticles is now a mature field. However, further advances, in particular in the field of \\ catalysis, require a more accurate description of their surface and of the transformations occurring beneath the surface in \\ the environment of use. Through a selection of case studies, this feature article proposes a journey from surface science to \\ nanoparticle design, while illustrating state-of-the-art spectroscopies that help provide a relevant description of inorganic \\ nanoparticles in the context of surface reactivity.
}

\section{Introduction: Surface reactivity of solids}

\section{Inorganic solids: core vs. surface}

Traditionally, chemists define solids through their composition and the local atomic arrangement (unit cell). This unit cell is then duplicated in the three directions of space to generate a quasi-infinite set of atoms. Inorganic solids can be amorphous (glasses) or crystalline. Solids can undergo reactions that change the local atomic arrangements (crystallization, amorphization, transformation of one phase into another one). They can also undergo reactions with incoming species (anions, cations, neutral atoms), eg. in the case of lithium insertion into metal oxides, or outgoing ones, eg. in the case of phase segregation. All these are encompassed in the field of "solid state chemistry", which is as old as chemistry itself and finds roots in metallurgy (Bronze Age, Iron Age, etc.) while it nourished the greatest hopes of Alchemy.

On the other hand, the chemist can choose to look at the few top layers of a solid (the surface). Composition, local arrangement and reactivity of this part of the solid can significantly differ from these of the core: this is the field of "surface sciences", with major achievements related to the development of scanning tunnelling electron microscopy. In this context, surface reactivity can be defined as the tendency of the surface to undergo a chemical reaction by itself or with an incoming atom or molecule. Because reactivity itself is deprived of an absolute sense, surface reactivity cannot be directly quantified, although thermodynamic and kinetic parameters, such as the energy released by a given reaction and its rate, inform on the reactivity of the surface.

Moreover, difficulty to provide an accurate description of the surface is acknowledged through a quote attributed to Linus Pauling: "God made the bulk; surfaces were invented by the devil". ${ }^{1}$ This complexity gave rise to a rich scientific domain, highlighted by the Nobel Prize of Gerhardt Ertl in 2007. Examples of surface-related phenomena are crystallographic reconstruction and phase segregation, spontaneous or as a result of the interaction with an adsorbate molecule. ${ }^{2,3,4}$
Figure 1 (left) illustrates spontaneous surface reconstruction of $\mathrm{Si}(100)$ in vacuum. ${ }^{2}$ The top layer of atoms forms a $\mathrm{p}(2 \times 1)$ structure (Figure $1 \mathrm{~A}$ ) that generates a corrugation of $0.31 \AA$ (Figure 1B). Surface reconstruction can also be triggered by the adsorption of a molecule like carbon monoxide, as illustrated on the $\mathrm{Pt}(100)$ surface (Figure $1 \mathrm{C}$ ). Combined DFT modeling and STM show the position of $\mathrm{CO}$ adsorbates and Pt atoms underneath (in light blue). ${ }^{4}$ In the starting geometry of the DFT model, the Pt atoms and $\mathrm{CO}$ molecules are separated by $0.37 \mathrm{~nm}$. After relaxation to minimize energy in the DFT calculation, the $\mathrm{CO}$ molecules form a $(3 \times 3)$ cluster with $\mathrm{O}-\mathrm{O}$ distances in the range $0.37-0.41 \mathrm{~nm}$, which matches the STM observations. The average Pt-Pt distance is similar to these of the bulk $(0.275 \mathrm{~nm})$, but side-view shows a significant corrugation of $1.10 \AA$ (Figure 1D).

Theses surface reconstructions have consequences on the mechanical properties (tribology) but also on the reactivity of the surface towards incoming molecules, hence in catalysis.

On top of surface reconstruction, surface of chemical compounds may undergo phase segregation as a form of surface reactivity, i.e. the surface may be enriched in one constituent. This was predicted and modelled theoretically for alloys, ${ }^{5}$ and largely described in the field of metallurgy. For instance, CuAu(100) is enriched in gold at its surface even at temperatures close to the melting temperature. ${ }^{6}$ Nanoscaled solids also may undergo surface segregation, as shown for PtFe alloy. ${ }^{7}$

In relation with catalysis, monitoring surface reconstruction and segregation is critical to the proper description of the active site. ${ }^{8}$ Recent breakthroughs in instrumentation, such as near-ambient pressure x-ray photoelectron spectroscopy, in situ scanning transmission electron microscopy or sum frequency generation spectroscopy, provided new insights to this challenge, as will be discussed in the following sections. Prior to this, it appears essential to define what a surface is and how the nanoscale influences this notion.

\footnotetext{
Sorbonne Université, CNRS, Collège de France, Laboratoire de Chimie de la Matière Condensée de Paris, 4 Place Jussieu, 75252 Paris, France. sophie.carenco@sorbonne-universite.fr

b. + Corresponding author.
} 
A

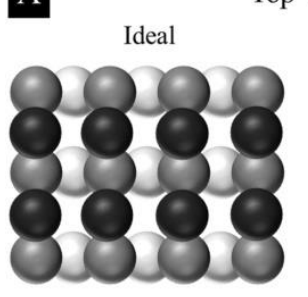

Top view

$\mathrm{p}(2 \times 1)$ reconstructed

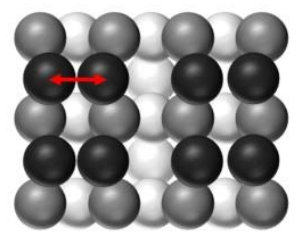

B

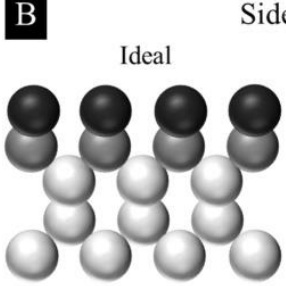

Side view

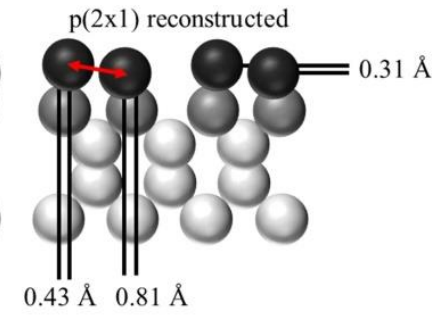

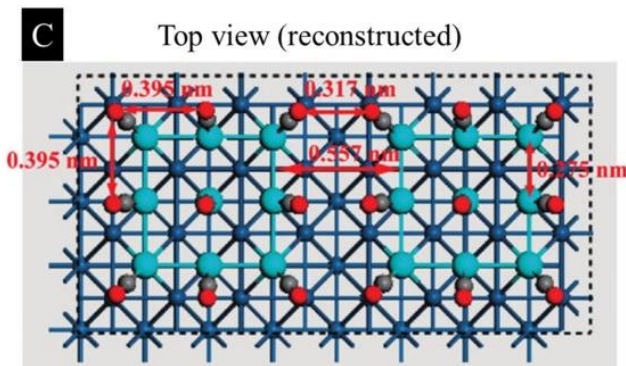

D

Side view (reconstructed)

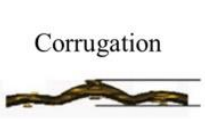

$1.10 \AA$

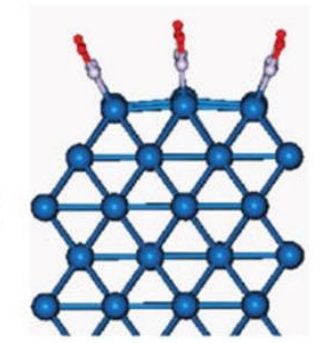

Figure 1: (A) Top view and (B) Side view of a Si(100) surface in ideal configuration (left) and after reconstruction in vacuum (right). Red arrows indicate the most displaced atoms after reconstruction, which extends 3 atomic layers into the bulk. (A) and (B) were redrawn from ref ${ }^{2}$. (C) Top view and (D) side view of the reconstruction of hex-Pt(100) as a consequence of carbon monoxide adsorption, according to DFT modeling. Corrugation is illustrated as an inset in (D). Pt in the slab is in dark blue, Pt at the surface in light blue, oxygen is in red and carbon in gray. (C) and (D): Adapted with permission from ref ${ }^{4}$. Copyright (2009) American Chemical Society.

\section{What is the effective thickness of the surface?}

A proper description of the surface starts with a simple question: what thickness, in number of atomic layers or any other relevant unit, should be considered as belonging to the surface?

(i) In geometric terms, it can be defined as the layer whose structure differs from these of the bulk. In this viewpoint, a solid that does not undergo surface reconstruction has a surface limited to the very last atomic layer, i.e. the one with dangling bonds, and the surface is only slightly thicker when reconstructed.

(ii) Another viewpoint consists in considering the electronic properties of the atoms: the surface is then defined through the band filling as a function of thickness. For example, for semi-conductors, band-bending is well described and depends on the nature of the junction, i.e. on the nature of the medium adjacent to the solid being considered.

(iii) A last viewpoint, which will be used in the following discussion, is based on the reactivity of the surface with the outside medium: in a chemical reaction limited to the surface (passivation, catalysis, etc.), the thickness of the surface is the region that is chemically reacting with incoming molecules $\left(\mathrm{O}_{2}\right.$, substrates, etc.). This empiric definition means that the surface thickness varies with the operating conditions (temperature, reaction time) and with the reaction considered. As an example, hydrogenation of unsaturated carbon-carbon bonds is comparatively described on Pt and Pd (Figure 2). On a hex$\mathrm{Pt}(100)$ surface (Figure 2A), adsorption of ethylene at room temperature results in the formation of ethylidyne and di- $\sigma$ bonded ethylene (from a pressure of 1.33 mbar). ${ }^{9}$ These species are observed by X-ray photoelectron spectroscopy (XPS). This does not modify the hex-Pt(100) structure, although adsorbates are mobile on the surface, with and without the presence of $\mathrm{H}_{2}$ in the gas mixture. ${ }^{10}$ In contrast with this, hydrogenation of acetylene on Pd leads to the formation of palladium carbide in the first sublayers of the metal (Figure 2B). ${ }^{11,12}$ Depending on the reaction conditions, Pd can incorporate large amounts of carbon distributed among the top metal interlayers, although the most stable configuration shows no surface carbon, according to DFT modeling. ${ }^{13}$

\section{How to monitor surfaces?}

The question discussed in the previous paragraph (thickness of the surface) is also related to the techniques that should be used to monitor the surface.

Traditional surface science toolbox encompasses scanning tunneling microscopy (STM) and low-energy electron diffraction (LEED). ${ }^{14}$ STM allows the cartography of the density of state of a surface (hence, its topology). Atomic resolution can be reached for a surface with low roughness (typically, a single-crystal). For crystalline surfaces, LEED informs on the crystallographic structure of the first layers. Like STM, it requires a flat surface to reach its full potential. Both techniques are thus not suited to the study of the surface of large nanoparticles in disordered arrays and/or supported on a secondary phase as typically found in heterogeneous catalysis. They are also not adequate for nanoparticles in solution (colloids). Hence, they will not be further discussed. Many alternatives are available to properly describe a nanoparticle surface $^{15}$ as discussed below. 

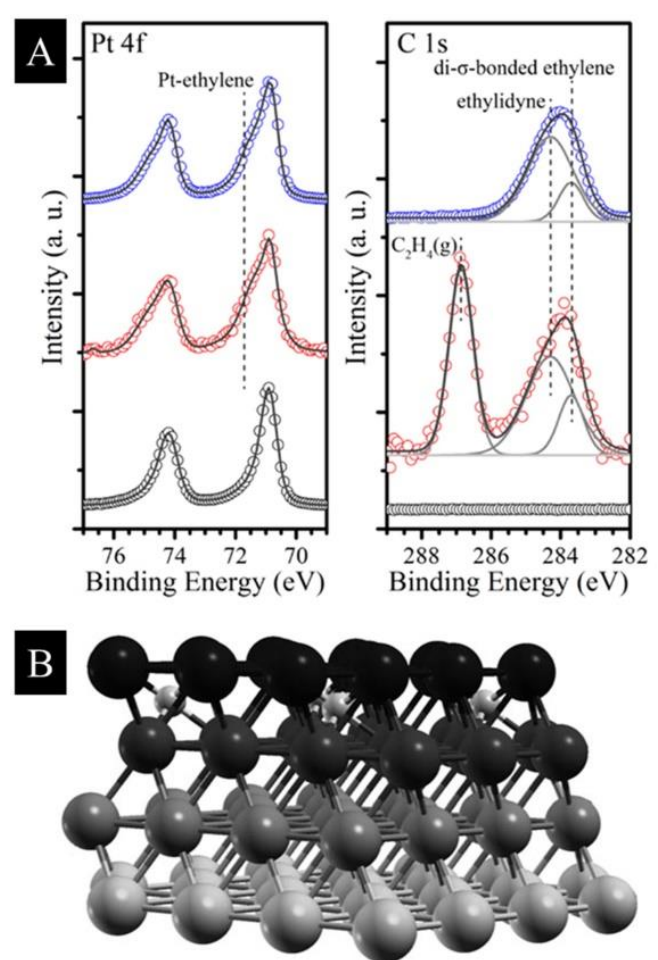

Figure 2: (A) Ethylene adsorption on hex-Pt(100) at room temperature. Adapted with permission from Ref ${ }^{9}$. Copyright (2013) American Chemical Society. (B) Model for the onset of formation of a surface carbide, eg. for $\mathrm{Pd}(111)$. Carbon atoms are the smaller spheres in light grey. Adapted with permission from Ref ${ }^{12}$. Copyright (C) 2010, John Wiley and Son.

Structure of nanoparticle's surface can be described by $x$-ray absorption spectroscopy (XAS) performed in total electron yield mode. In this mode, XAS is measured using the secondary electrons emitted from the solid. These come from the near surface because of the reduced inelastic mean free path (IMFP) in solids, typically in the $\mathrm{nm}$ range. ${ }^{16}$ L-edges of firstraw transition metals such as copper and cobalt, ${ }^{17,18}$ and $\mathrm{K}$ edges of light elements such as oxygen, ${ }^{19,20,21}$ were investigated with this method. X-ray absorption near edge structure (XANES) informs on the local environment of the elements in the compound. Its feasibility was demonstrated on single crystals, but also on foils and on nanoparticles, from ultra-high vacuum (UHV) to a pressure of 1 bar, thus bridging both the so-called "material gap" and "pressure gap".

Photoelectrons can also be collected in an analyzer that measures their kinetic energy: this is $\mathrm{x}$-ray photoemission spectroscopy (XPS), a technique intrinsically suited for surface analysis. Interestingly, varying the photon energy of the beam (or alternatively its incidence angle) allows probing the surface at different depths, from less than $1 \mathrm{~nm}$ to a few $\mathrm{nm}$. This depth is evaluated using models that simulate the IMFP for the electron considered in a chosen element. ${ }^{16}$ This is exemplified in Figure $3 \mathrm{~A}$, which describes the $\mathrm{Pt} 4 \mathrm{f}$ XPS of CoPt nanoparticles as a function of photon beam energy. By measuring a series of spectra, a model was proposed for the distribution of $\mathrm{Co}$ and $\mathrm{Pt}$ within the nanoparticle inorganic core (Figure 3B).
A Pt $4 \mathrm{f}$ (X-ray photoelectron spectropscopy)
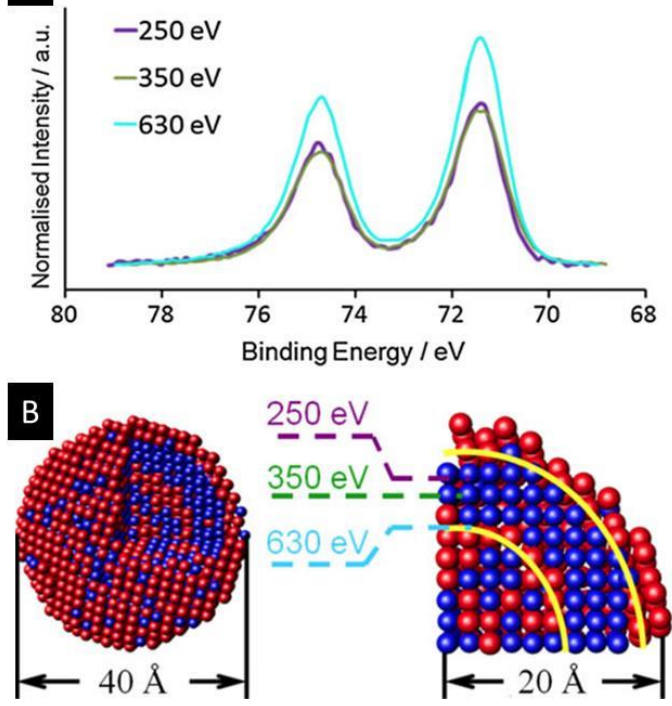

Figure 3: (A) Pt $4 \mathrm{f} X P$ spectra of $4 \mathrm{~nm}$ CoPt nanoparticles, collected with photon beam energy of 250,350 and $630 \mathrm{eV}$, corresponding to probing depths of $0.48,0.58$ and $0.90 \mathrm{~nm}$. (B) Proposed model: a 7-layer nanoparticle with Co (in blue) and Pt (in red) atoms unevenly distributed. Top layer is richer in Pt. Adapted with permission from Ref ${ }^{20}$. Copyright (c) 2011, Springer Nature.

As a complement to X-ray spectroscopies, vibrational spectroscopies (infra-red, Raman, etc.) inform not only on the inorganic compounds but also on the nature of organic adsorbate. Some of them are surface-sensitive by design. This is the case of sum frequency generation spectroscopy (SFG), which is sensitive to a monolayer of adsorbates. It was for example used to investigate the influence of poly(vinylpyrrolidone) (PVP) capping agent on Pt nanoparticles used for ethylene hydrogenation. ${ }^{22}$ Vibrational spectroscopies will not be further discussed here but they also include broadly-used diffuse reflectance infrared Fourier transform spectroscopy (DRIFTS). However, they raise the question of the nature of the chemical bonds between adsorbates (capping ligand, reactant) and surface. Adsorbates are modifying the electronic properties of the surface, as explained by $R$. Hoffmann in a review published in $1988 .^{23}$

Because nanoparticles exhibit a comparatively high amount of surface compared with macroscopic compound, the next section is dedicated to showing if and how nanoparticle's surface reactivity differs from these of a macroscopic solid.

\section{Is a nano-object special in terms of surface reactivity?}

Nanoparticles exhibit a high surface-to-volume ratio, compared with macroscopic compounds. As a consequence, surface energy becomes significant vs. lattice energy. This has a direct consequence on the shape adopted by a crystalline nanoparticle: under thermodynamic control, the facet with lower surface energy will be favored, and the overall shape will minimize the total surface energy (Wulff construction). For instance, based on theoretical modeling, Barnard proposed a phase diagram for the shape of palladium nanocatalysts. ${ }^{24}$ Beyond these geometric considerations, electronic properties, 
hence surface reactivity, may vary with the number of atoms of the nano-object.

Influence of nanoparticle's diameter on the catalytic properties

The influence on diameter on the activity of gold nanoparticles for the oxidation of carbon monoxide is a seminal example of size-effect in catalysis. ${ }^{25,26} \mathrm{CO}$ adsorption on surfaces on metal is well described, in particular with the help of modelling, ${ }^{27}$ but composition and size effects on the surface reactivity are being scrutinized in order to improve chemical and electrochemical reduction performances and selectivity. ${ }^{28,29}$

Recently, the diameter of cobalt nanoparticles was correlated with their turn-over frequency (TOF) for the reduction of carbon monoxide by $\mathrm{H}_{2} \cdot{ }^{30}$ Smaller nanoparticles (diameter below $5 \mathrm{~nm}$ ) were found to be less active than larger ones $(10 \mathrm{~nm})$, and above $15 \mathrm{~nm}$ a plateau was observed. In situ x-ray absorption (XAS) at Co L-edge confirmed the metallic state of cobalt under 1 bar of $\mathrm{H}_{2}$ at $250^{\circ} \mathrm{C} .^{21} \mathrm{CO}$ dissociation was followed by the appearance of a cobalt oxide peak on the $\mathrm{O} \mathrm{K}$ edge spectrum. It was quantified and compared for 4,10 and $15 \mathrm{~nm}$ cobalt nanoparticles (Figure 4, top). The trend was similar to these observed for the TOF: larger nanoparticles showed more $\mathrm{CO}$ dissociation than smaller ones, and this effect was more pronounced when the temperature was higher (Figure 4, bottom).
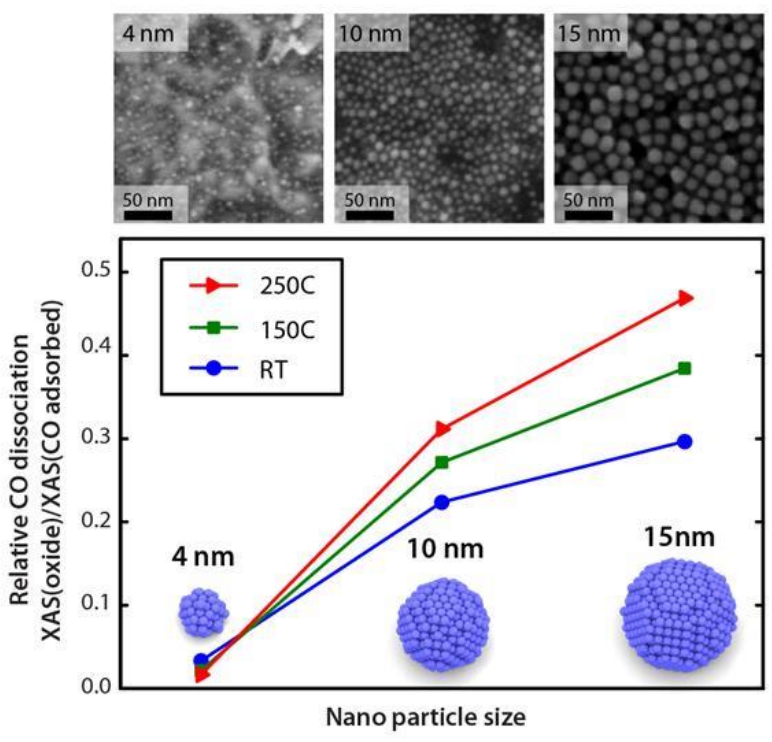

Figure 4: (Top) Scanning electron microscopy of the 4, 10 and $15 \mathrm{~nm}$ cobalt nanoparticles. (Bottom) Relative concentration of dissociated $\mathrm{CO}$ species on nanoparticles after exposure to $\mathrm{CO} / \mathrm{He}$ at different temperatures. The concentration is calculated from the area of the oxide XAS peak relative to the $\pi^{*}$ peak from intact adsorbed CO. Adapted from Ref ${ }^{21}$.

Size-effect for nanocatalysts is being extensively studied for different metals (eg. $\mathrm{Ru}^{31}$ ) and reactions (eg. oxygen reduction reaction ${ }^{32}$ ) because it helps adjusting the surface reactivity. This can be done only when nanoparticles survive to the presence of reactive gases. For many inorganic compounds traditionally prepared as macroscopic crystals, description of the surface reactivity, for instance upon exposure to air, is not available.

\section{Controlling surface reactivity for preserving the nanoparticles} composition and structure

Surface oxidation of non-noble metal and alloy nanoparticles (eg. iron, ${ }^{33}$ nickel-cobalt ${ }^{34}$ ) is a well-known process. However, other compounds were not much explored. This is the case of lanthanide oxysulfides of formula $\operatorname{Ln}_{2} \mathrm{O}_{2} \mathrm{~S}$ ( $\mathrm{Ln}=$ lanthanide), which exhibit a lamellar structure with $\left[\mathrm{Ln}_{2} \mathrm{O}_{2}\right]^{2+}$ and $\mathrm{S}^{2-}$ planes (Figure 5A). The structure was reported in $1947^{35}$ and the compounds were prepared for all Ln except promethium. ${ }^{36}$ However, the first $\mathrm{Ln}_{2} \mathrm{O}_{2} \mathrm{~S}$ nanoparticles were reported as late as $2000 .^{37}$ They found applications in the fields of upconversion, persistent luminescence and magnetic contrast agent for MRI. Few examples deal with catalysis, eg. oxygen reduction reaction ${ }^{38}$ and water-gas shift. ${ }^{39}$

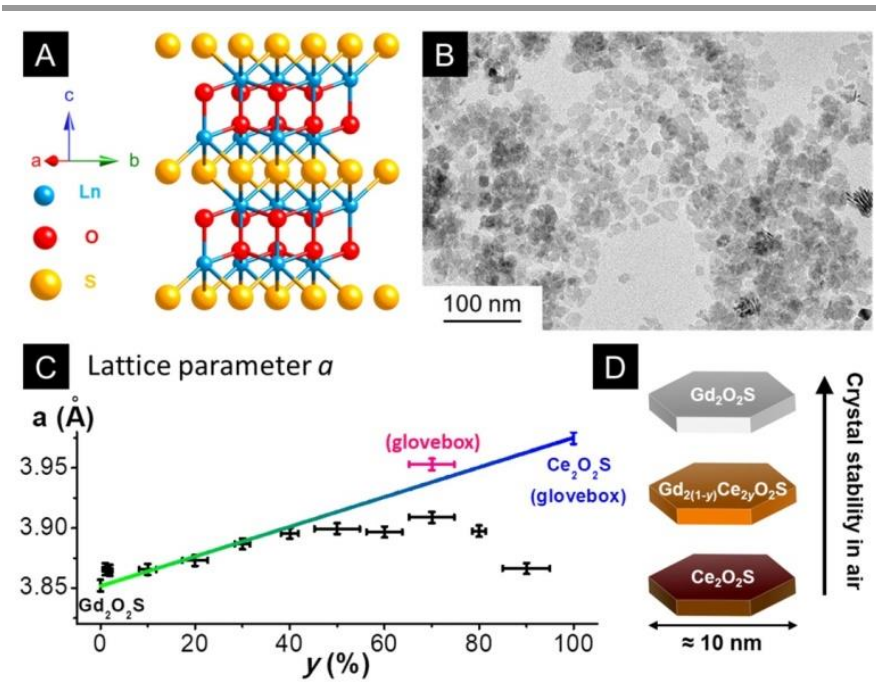

Figure 5: (A) $\mathrm{Ln}_{2} \mathrm{O}_{2} \mathrm{~S}$ hexagonal crystal structure (for $\mathrm{Ln}=\mathrm{Gd}$, see JCPDS 06-8819). (B) TEM of $\mathrm{Gd}_{2(1-y)} \mathrm{Ce}_{2 y} \mathrm{O}_{2} \mathrm{~S}$ nanoparticles containing $37.5 \%$ Ce among total Ln, (C) Lattice parameter $a$ as a function of $y$ in $\mathrm{Gd}_{2(1-y)} \mathrm{Ce}_{2 y} \mathrm{O}_{2} \mathrm{~S}$, extracted from X-ray diffractograms on powders $\left(\right.$ see ${ }^{40}$ ). The line is a guide to the eye indicating the theoretical value for $a$, according to Vegard's law. All powders were isolated and measured under air except the two samples marked "glovebox". (D) Trend for crystal stability in air.

The surface state of metal oxysulfides remains mostly undescribed, although one can expect sulfide species to undergo oxidation when exposed to $\mathrm{O}_{2}$ and/or $\mathrm{H}_{2} \mathrm{O}$. Recently, we studied the formation of $\mathrm{Gd}_{2(1-y)} \mathrm{Ce}_{2 y} \mathrm{O}_{2} \mathrm{~S}$ nanoparticles with $y$ varying from 0 to 1 (Figure $5 \mathrm{~B}$ ). ${ }^{40} \quad \mathrm{Ce}_{2} \mathrm{O}_{2} \mathrm{~S}$, containing exclusively $\mathrm{Ce}^{(\mathrm{III})}$, was found to be highly air-sensitive and had to be isolated in an inert glovebox. In air, the formation of $\mathrm{CeO}_{2}$ (containing $\mathrm{Ce}^{(\mathrm{IV})}$ ) was observed within days from x-ray diffraction (XRD) on the nanoparticles powder. ${ }^{40}$ Solid solution containing both cerium and gadolinium cations presented a lattice parameter $a$ that followed Vegard's law (Figure 5C), for $y<40 \%$. Nanoparticles with high Ce content $(y>80 \%)$ were rapidly degraded in air. For intermediate $y$ values, a plateau was observed in terms of lattice parameter. Comparison with a sample isolated and measured under inert atmosphere for $y=70 \%$ revealed the effect of air on the lattice parameter $a$ : partial degradation of the nanoparticles occurred due to reaction with humidity and $\mathrm{O}_{2}$. At this stage, the structural discussion seemed to indicate that higher cerium fraction was responsible for the sensitivity to air (Figure 5D) and that cerium-free $\mathrm{Gd}_{2} \mathrm{O}_{2} \mathrm{~S}$ was not affected by exposure to air. 
The absence of oxidized sulfur $\left(S^{(I V)}\right.$ and $\left.S^{(V I)}\right)$ in the powders isolated under inert atmosphere was confirmed by XAS at sulfur $\mathrm{K}$-edge on $\mathrm{Gd}_{2} \mathrm{O}_{2} \mathrm{~S}$ and $\mathrm{Ce}_{2} \mathrm{O}_{2} \mathrm{~S}$ (Figure 6). However, $\mathrm{Gd}_{2} \mathrm{O}_{2} \mathrm{~S}$ powder exposed to air showed the presence of oxidized sulfur. XPS confirmed the presence of sulfonates and sulfates. Sulfur oxidation was driven by the formation of highly stable S-O bonds.

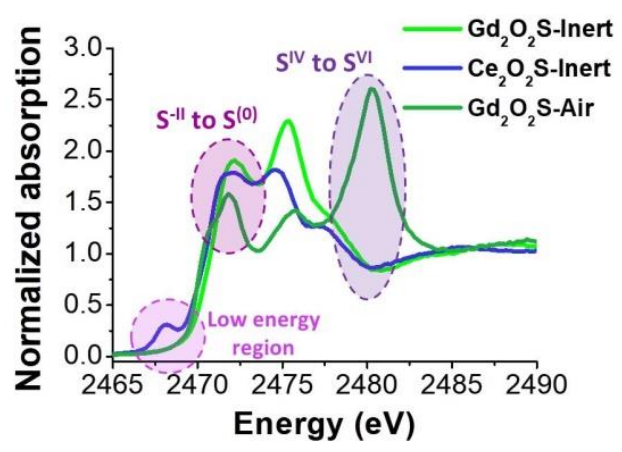

Figure 6: X-ray absorption spectra at Sulfur K-edge on three pellets of nanoparticles: $\mathrm{Gd}_{2} \mathrm{O}_{2} \mathrm{~S}$ (dark green) and $\mathrm{Ce}_{2} \mathrm{O}_{2} \mathrm{~S}$ (blue) isolated and stored under inert atmosphere, $\mathrm{Gd}_{2} \mathrm{O}_{2} \mathrm{~S}$ exposed to air for several days (light green). Dotted circles are a guide to the eye.

This example illustrates that structures considered as nonreactive at the macroscopic scale or even at the nano-scale (based on XRD) may actually be the theatre of a significant surface reactivity. Here, the nanoparticles at stake present a thickness of a few layers only: partial surface oxidation already represent a significant change of the overall chemical object, with consequences to be expected on the catalytic properties.

\section{Surface reactivity inducing in-depth restructuring of nanoparticles}

Beyond surface oxidation, state-of-the-art in situ spectroscopy and microscopy now allow monitoring the state of nanoparticles surface exposed to reactive gases, eg. while simulating the Fisher-Tropsch process, the methanation of $\mathrm{CO}_{2}$, or other reactions relevant to the industry. Surface restructuring as a consequence of $\mathrm{CO}$ adsorption is a welldescribed process for flat surfaces, such as single-crystals. ${ }^{41}$ Following the same process, nanoparticles transformation in terms of morphology or even composition can be drastic, as illustrated for nanoparticles of $\mathrm{Rh},{ }^{42} \mathrm{RhPd}$ and $\mathrm{PdPt},{ }^{43,44}$ or PtCo, ${ }^{45,20}$ for instance.

A typical methodology to study these restructuring is to synthesize well-defined nanoparticles from colloidal synthesis in organic solvents. The nanoparticles are stabilized by organic ligands (eg. oleates), which are burnt by a treatment in $\mathrm{O}_{2}$ at ca $200{ }^{\circ} \mathrm{C}$. The metallic state of the nanoparticles is restored by a second treatment under $\mathrm{H}_{2}$ in the same temperature range, and to improve the process this is repeated a couple of times. The nanoparticles are then exposed to model reaction conditions (gas mixture) and heated to the desired temperature.

Under these conditions, a core-shell structure may rearrange into a hollow one due to the diffusion of metals from the core to the outside of the nanoparticles. This is what was experimentally observed with copper-cobalt nanoparticles exposed to a mixture of carbon monoxide and hydrogen at
$250{ }^{\circ} \mathrm{C}$ (Figure 7A). In this case, the lack of miscibility of copper and cobalt drove the segregation of a minority of the copper outside of the initial nanoparticles, which formed new hollow particles. ${ }^{46}$ This was revealed by combining ex situ microscopy and EDS mapping with in situ near-ambient-pressure XPS.

Cobalt and nickel are, by contrast, more miscible, which may be the driving force for the formation of an alloy upon heat transfer. After cycles of oxidation and reduction, core-shell nickel-cobalt nanoparticles were found to rearrange as partially hollow nanoparticles, with a shell of NiCo alloy and a nickel-rich core (Figure 7B). ${ }^{47}$ They did not further evolve under the mixture of $\mathrm{CO}_{2}$ and $\mathrm{H}_{2}$.

А

Cycles of oxidation and reduction, then exposure to $\mathrm{CO}+\mathrm{H}_{2}$
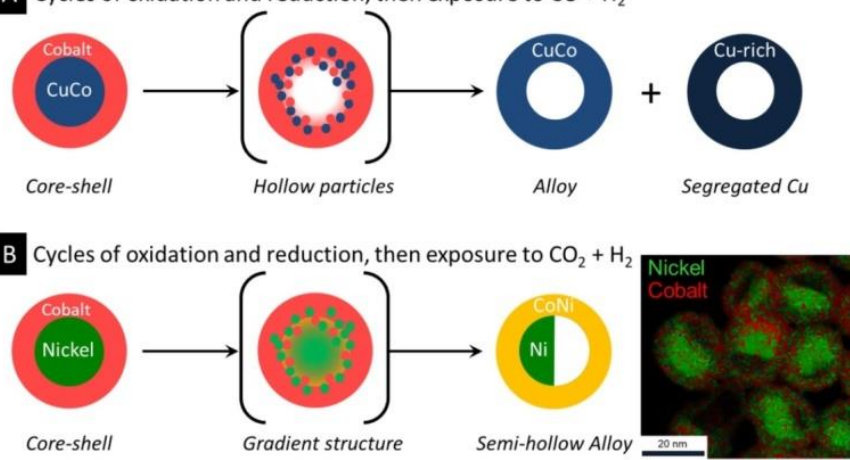

Figure 7: Bimetallic nanoparticles exposed to reactive gases: (A) copper-cobalt nanoparticles, ${ }^{46}$ (B) nickel-cobalt nanoparticles (with EDS mapping on the right). ${ }^{47}$

This pair of examples illustrates the diversity of scenarios that are encountered. Under reactive gases, none of the two systems exhibited the catalytic surface that one would have expected on paper. This highlights the importance of an accurate description of the nanoparticles in relation with the reaction being investigated. The driving forces for these transformations are manifold and potentially antagonists: coordination strength of the incoming molecule, diffusion coefficient in the inorganic core, relative surface energy of the metals, etc. While it is essential to study model systems (welldefined nanoparticles) to understand them, real catalysts should also be analyzed, in the presence of the catalysts support. This was done for example for $\mathrm{Cu} / \mathrm{ZnO}$ on $\mathrm{Al}_{2} \mathrm{O}_{3}{ }^{48}$ and for PtCo on $\mathrm{TiO}_{2}{ }^{49}$ Technical development in analytical microscopy and spectroscopy is at the heart of progress in this field. ${ }^{50,51,8,52,53}$

So far, the inorganic core was the focus of the discussion. As briefly mentioned above, nanoparticles may be covered by organic ligands (also called surfactants or capping agents, depending of the context). Instead of burning them away as in the previous examples, these ligands can be used to tune the surface properties of the nanoparticles. This is the object of the next section.

\section{Ligand-covered nanoparticles}

\section{Ligands as reactive species}

In most cases, ligands such as polyvinylpyrolidone (PVP), alkylamines or alkylphosphines, are introduced during the synthesis of the nanoparticles in order to control their size and 
morphology, as extensively reviewed in the literature. ${ }^{54,55}$ Their interaction with the metal precursors and/or with the nanoparticle surface are critical to the nucleation and growth stages of the synthesis. ${ }^{56}$

Although they may be washed or destroyed (partially or quantitatively) during the purification step, they are often not innocent regarding the nature of the nanoparticles surface: first, because they contributed to select the facets exhibited by the nanoparticles, second, because their degradation process is rarely complete and controlled. For instance, tri- $n$ octylphosphine (TOP) decomposes at much lower temperature $\left(150^{\circ} \mathrm{C}\right)$ onto a nickel nanoparticle surface than as a free species. $^{57}$ As a consequence of ligand decomposition, phosphorus contamination was observed in several cases with consequences on the activity and selectivity of the nanoparticles in heterogeneous catalysis. ${ }^{20,47}$

Taking advantage of this, ligand decomposition can be exploited to produce an organic carbon shell around nanoparticles at $400{ }^{\circ} \mathrm{C}$ under inert atmosphere, thus increasing the conductivity of the powder. This was illustrated with $\mathrm{Ni}_{2} \mathrm{P}$ nanoparticles used as negative electrode in lithium batteries: carbon-covered nanoparticles showed better $\mathrm{Li}$ uptake by providing a Li-permeable electron-conductive percolating network and the electrode had a better mechanical stability. ${ }^{58}$ One step further, ligands can be used to transform metal nanoparticles into metal carbides and metal phosphides, as was done on nickel nanoparticles, among others. ${ }^{59,60}$

On the other hand, an alternative approach is to select ligands that survive the reactivity studies and become an ally to control the nanoparticle's surface reactivity.

Ligands tuning the electronic properties of the surface

Ligand chemisorption on a surface can be described with a frontier orbital approach: depending on the relative energy of the orbitals and the surface Fermi level, as well as on the quality of the overlap, charge transfer from one to the other may occur. ${ }^{23}$ As a consequence, both surface and ligand will be affected. $^{61}$

In two separate studies, ruthenium nanocatalysts were characterized by XPS. In the first case, the nanoparticles came from a ligand-free sol-gel reaction and were deposited on a $\mathrm{TiO}_{2}$ support: they can be regarded as "bare nanoparticles" (Figure $8 \mathrm{~A}$ ). ${ }^{62}$ After the reduction step, XPS of Ru $3 d_{5 / 2}$ showed a main peak at a binding energy of $280.0 \mathrm{eV}$, similar to these of bulk ruthenium metal (Figure $8 \mathrm{~A}$, indicated by a red dotted line). This metallic state persisted under $\mathrm{CO}_{2}$ methanation conditions at $200^{\circ} \mathrm{C}$. In the second case, ruthenium nanoparticles were prepared by the decomposition of a $\mathrm{Ru}(0)$ molecular precursor and stabilized in solution with a bidendate phosphine (dppb=1,4-bis(diphenylphosphino)butane), as shown on Figure $8 \mathrm{~B} .{ }^{63}$ The nanoparticles were, as expected, highly air-sensitive and active for $\mathrm{CO}$ hydrogenation in solution, but were deposited on a gold substrate in order to measure XPS. Fresh nanoparticles showed a binding energy of $280.5 \mathrm{eV}$ for Ru $3 \mathrm{~d}_{5 / 2}$ (Figure $8 \mathrm{~B}$ ), higher than the bare nanoparticles. This suggested that the phosphine was overall depleting the nanoparticles surface in electrons. This state mostly survived after introduction of a mixture of $\mathrm{CO}$ and $\mathrm{H}_{2}$, although partial $\mathrm{CO}$ dissociation was observed and resulted in the formation of a second Ru species, more oxidized. Both types of nanoparticles (bare and ligand-covered) are active for the related $\mathrm{CO}$ and $\mathrm{CO}_{2}$ hydrogenation reactions. In the second case, the ligand was shown to improve the selectivity for $\mathrm{C}_{2}-\mathrm{C}_{4}$ products, thus modulating the intrinsic selectivity of the Ru surface.

The recent literature now contains many examples of ligandcontrolled catalytic reactions, including enantioselective ones. ${ }^{64,65,66}$ This field is rapidly expanding, at the cross-roads of advanced nanoparticles synthesis, spectroscopies that monitor the surface state in situ, and a growing interest for the catalytic formation of higher added-values molecules (eg. longchain alcohols, chiral compounds) from cheap carbon sources $\left(\mathrm{eg}, \mathrm{CO}_{2}\right)$. 
A Bare nanoparticles
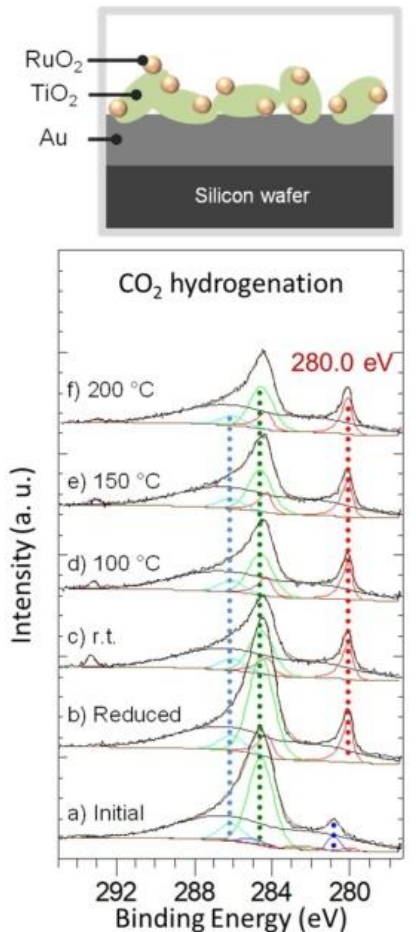

B

Ligand-covered nanoparticles
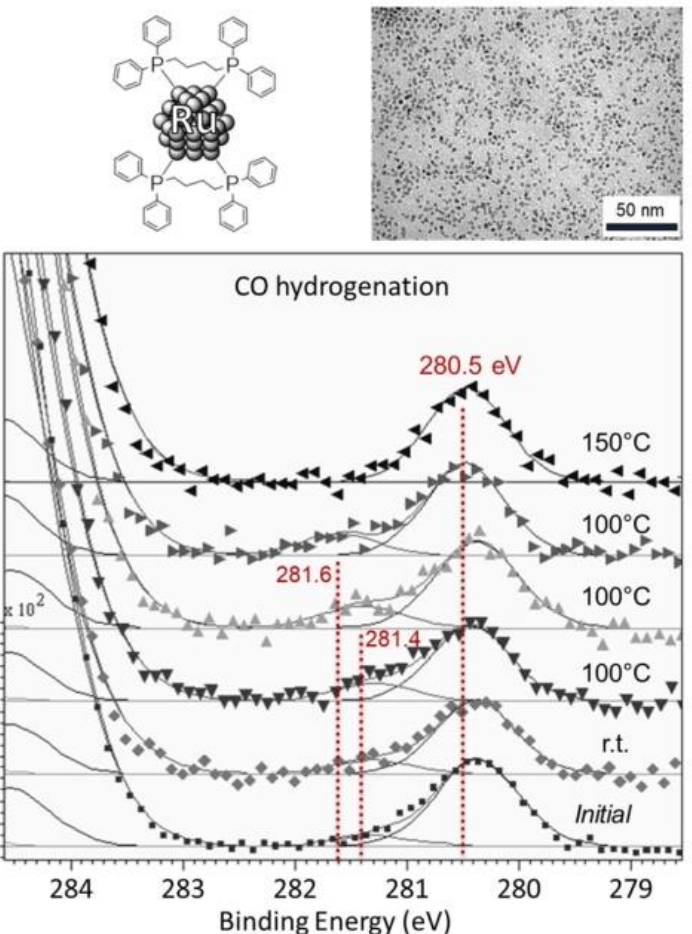

Figure 8: (A) Bare Ru nanoparticles on $\mathrm{TiO}_{2}$, active for $\mathrm{CO}_{2}$ methanation. Bottom: XPS of the fresh sample (before reduction of the Ru), of the reduced sample (showing metallic Ru), and during the catalytic reaction under a mixture of $\mathrm{CO}_{2}$ and $\mathrm{H}_{2}{ }^{62}$ (B) Phosphine-covered ruthenium nanoparticles prepared and used in solutions. Bottom: XPS of the nanoparticles deposited on a gold substrate: fresh sample, and sample during $\mathrm{CO}$ hydrogenation reaction. Dotted lines are a guide to the eye.

\section{Summary and Outlooks}

In this feature article, the interplay between adsorbates and surfaces was discussed, for macroscopic solids but also for nanoparticles. This highlights the importance of clarifying the nature of ligands present on nanoparticles surface, but also the necessity to properly follow the transformations of their inorganic core in the environment of use (ambient air for devices, under gas mixture for catalysis, etc.). Only in these conditions will the description of a nanoparticle provide accurate understanding of its reactivity.

Furthermore, ligands offer the opportunity to tune this reactivity, in particular for catalysis purposes. Promising outlooks of this field can be delineated in two steps:

(1) the fabrication of "bare" or ligand-free nanoparticles and clusters from innovative synthetic routes, combining the advantages of heterogeneous catalysts preparation (no solvent, no organic stabilizer) with the excellent morphology and composition control available in colloidal synthesis. ${ }^{67,68,69}$

(2) the remodeling of their surface reactivity using selected ligands with peculiar coordinating modes, eg. providing Lewis acido-basic interaction at the surface. ${ }^{70,71}$

Original synthetic approaches, forming nanoparticles with unprecedented composition and/or exposed surfaces, should be driven and combined with the most recent modelling strategy, such as the automated construction of surface phase diagrams, ${ }^{72}$ in order to target systems with relevant properties in a given catalytic process. However, in-depth understanding and improvement of proof-of-concept nanomaterials will still require more powerful and multispectral analytical tools (eg. simultaneous XAS, XRD and IR), as currently being developed on dedicated beamlines worldwide.

\section{Conflicts of interest}

There are no conflicts to declare.

\section{Acknowledgements}

Sorbonne Université, CNRS and Collège de France are acknowledged. S.C. gratefully acknowledges co-authors of the works described in this Feature Article. This project has received funding from the European Research Council (ERC) under the European Union's Horizon 2020 research and innovation programme (grant agreement No 758480).

\section{Author biography}

Sophie Carenco graduated in 2008 from Ecole Polytechnique, in France. She obtained her PhD in 2011 from Sorbonne Université-UPMC, Paris, for her work on the synthesis and applications of metal phosphide nanoparticles. From 2012 to 
2013, she was a post-doctoral fellow at Lawrence Berkeley National Lab, Berkeley, California, where she used synchrotron-based in situ spectroscopies to monitor the surface state of metal nanoparticles during model catalytic reactions. In 2014, she joined CNRS as a researcher in the Materials Science department of Sorbonne Université, Paris. She works on developing novel synthetic routes for nanoparticles (metals, metal phosphides, metal oxysulfides, metal carbides) of controlled composition and surface state, with applications in reactivity and catalysis. She was awarded the European Young Chemist Award in 2010, the L'OrealUNESCO Fellowship in 2014, the Bronze Medal of CNRS in 2018 and an ERC Starting Grant in 2017. She is also involved in scientific outreach and published in 2012 a book about nanomaterials and chemistry.

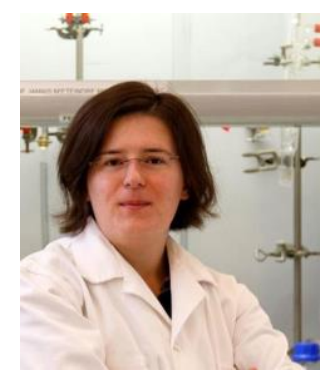

\section{Table of Content Entry}

Surface and core of inorganic nanoparticles may undergo profound transformations in their environment of use. Accurate description is key to understand and control surface reactivity.

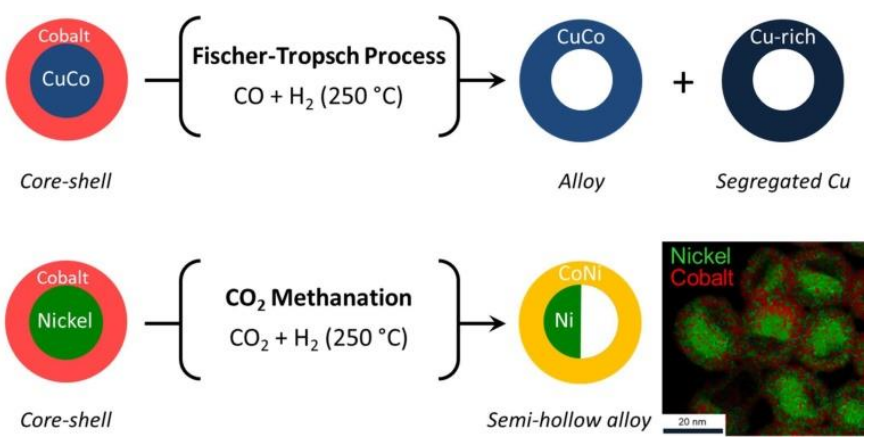

\section{Notes and references}

(1) Jamtveit, B.; Meakin, P. Growth, Dissolution and Pattern Formation in Geosystems; Springer, 1999.

(2) Somorjai, G. A. Surface Reconstruction and Catalysis. Annu. Rev. Phys. Chem. 1994, 45, 721-751.

(3) Tao, F.; Dag, S.; Wang, L.-W.; Liu, Z.; Butcher, D. R.; Bluhm, H.; Salmeron, M.; Somorjai, G. A. Break-up of Stepped Platinum Catalyst Surfaces by High CO Coverage. Science 2010, 327, 850853.

(4) Tao, F.; Dag, S.; Wang, L.-W.; Liu, Z.; Butcher, D. R.; Salmeron, M.; Somorjai, G. a. Restructuring of Hex-Pt(100) under CO Gas Environments: Formation of 2-D Nanoclusters. Nano Lett. 2009, 9, 2167-2171.

(5) Burton, J. Surface Segregation in Alloys. J. Catal. 1975, 37, 106-
113.

(6) Beikler, R.; Taglauer, E. Surface Segregation at the Binary Alloy CuAu (100) Studied by Low-Energy lon Scattering. Surf. Sci. 2016, 643, 138-141.

(7) Prabhudev, S.; Bugnet, M.; Zhu, G.-Z.; Bock, C.; Botton, G. A. Surface Segregation of Fe in Pt-Fe Alloy Nanoparticles: Its Precedence and Effect on the Ordered-Phase Evolution during Thermal Annealing. Chem CatChem 2015, 7, 3655-3664.

(8) Zafeiratos, S.; Piccinin, S.; Teschner, D. Alloys in Catalysis: Phase Separation and Surface Segregation Phenomena in Response to the Reactive Environment. Catal. Sci. Technol. 2012, 2, 1787.

(9) Zhu, Z.; Butcher, D. R.; Mao, B.; Liu, Z.; Salmeron, M.; Somorjai, G. A. In Situ Scanning Tunneling Microscopy and X-Ray Photoelectron Spectroscopy Studies of Ethylene-Induced Structural Changes on the Pt(100)-Hex Surface. J. Phys. Chem. C 2013, 117, 2799-2804. Butcher, D. R.; Zhu, Z.; Mao, B.; Wang, H.; Liu, Z.; Salmeron, M.; Somorjai, G. a. Mobility on the Reconstructed Pt(100)-Hex Surface in Ethylene and in Its Mixture with Hydrogen and Carbon Monoxide. Chem. Commun. (Camb). 2013, 49, 6903-6905. Teschner, D.; Révay, Z.; Borsodi, J.; Hävecker, M.; Knop-Gericke, A.; Schlögl, R.; Milroy, D.; Jackson, S. D.; Torres, D.; Sautet, P. Understanding Palladium Hydrogenation Catalysts: When the Nature of the Reactive Molecule Controls the Nature of the Catalyst Active Phase. Angew. Chem. Int. Ed. Engl. 2008, 47, 92749278.

Sautet, P.; Cinquini, F. Surface of Metallic Catalysts under a Pressure of Hydrocarbon Molecules: Metal or Carbide? ChemCatChem 2010, 2, 636-639.

Torres, D.; Cinquini, F.; Sautet, P. Pressure and Temperature Effects on the Formation of a Pd/C Surface Carbide: Insights into the Role of $\mathrm{Pd} / \mathrm{C}$ as a Selective Catalytic State for the Partial Hydrogenation of Acetylene. J. Phys. Chem. C 2013, 117, 1105911065.

Evans, C.; Brundle, R.; Wilson, S. Encyclopedia of Materials Characterization; Elsevier, Ed.; 1992.

Baer, D. R.; Engelhard, M. H.; Johnson, G. E.; Laskin, J.; Lai, J.; Mueller, K.; Munusamy, P.; Thevuthasan, S.; Wang, H.; Washton, N.; et al. Surface Characterization of Nanomaterials and Nanoparticles: Important Needs and Challenging Opportunities. J. Vac. Sci. Technol. A Vacuum, Surfaces, Film. 2013, 31, 50820. Tanuma, S.; Powell, C. J.; Penn, D. R. Calculation of Electron Inelastic Mean Free Paths (IMFPs) VII. Reliability of the TPP-2M IMFP Predictive Equation. Surf. Interface Anal. 2003, 35, 268-275. Jiang, P.; Prendergast, D.; Borondics, F.; Porsgaard, S.; Giovanetti, L.; Pach, E.; Newberg, J.; Bluhm, H.; Besenbacher, F.; Salmeron, M. Experimental and Theoretical Investigation of the Electronic Structure of $\mathrm{Cu}(2) \mathrm{O}$ and $\mathrm{CuO}$ Thin Films on $\mathrm{Cu}(110)$ Using X-Ray Photoelectron and Absorption Spectroscopy. J. Chem. Phys. 2013, 138, 24704.

(18) Zhang, H.; Wang, W.-C.; Glans, P.-A.; Liu, Y.-S.; Kapilashrami, M.; Chen, J.-L.; Chang, C.; Salmeron, M.; Escudero, C.; Pach, E.; et al. Developing Soft X-Ray Spectroscopy for in Situ Characterization of Nanocatalysts in Catalytic Reactions. J. Electron Spectros. Relat. Phenomena 2014, 197, 118-123.

Zheng, F.; Alayoglu, S.; Pushkarev, V. V.; Beaumont, S. K.; Specht, C.; Aksoy, F.; Liu, Z.; Guo, J.; Somorjai, G. a. In Situ Study of Oxidation States and Structure of $4 \mathrm{~nm}$ CoPt Bimetallic Nanoparticles during CO Oxidation Using X-Ray Spectroscopies in 
Comparison with Reaction Turnover Frequency. Catal. Today 2012, 182, 54-59.

(20) Alayoglu, S.; Beaumont, S. K.; Zheng, F.; Pushkarev, V. V.; Zheng, H.; lablokov, V.; Liu, Z.; Guo, J.; Kruse, N.; Somorjai, G. a. CO2 Hydrogenation Studies on Co and CoPt Bimetallic Nanoparticles Under Reaction Conditions Using TEM, XPS and NEXAFS. Top. Catal. 2011, 54, 778-785.

(21) Tuxen, A.; Carenco, S.; Chintapalli, M.; Chuang, C.-H.; Escudero, C.; Pach, E.; Jiang, P.; Borondics, F.; Beberwyck, B.; Alivisatos, A. P.; et al. Size-Dependent Dissociation of Carbon Monoxide on Cobalt Nanoparticles. J. Am. Chem. Soc. 2013, 135, 2273-2278. Krier, J. M.; Michalak, W. D.; Baker, L. R.; An, K.; Komvopoulos, K.; Somorjai, G. A. Sum Frequency Generation Vibrational Spectroscopy of Colloidal Platinum Nanoparticle Catalysts: Disordering versus Removal of Organic Capping. J. Phys. Chem. C 2012, 116, 17540-17546.

(23) Hoffmann, R. A Chemical and Theoretical Way to Look at Bonding on Surfaces. Rev. Mod. Phys. 1988, 60, 601-628.

(24) Barnard, A. S. Mapping the Shape and Phase of Palladium Nanocatalysts. Catal. Sci. Technol. 2012, 2, 1485.

(25) Haruta, M.; Kobayashi, T.; Sano, H.; Yamada, N. Novel Gold Catalysts for the Oxidation of Carbon Monoxide at a Temperature Far below 0.DEG.C. Chem. Lett. 1987, 405-408.

(26) Shaikhutdinov, S. K.; Meyer, R.; Naschitzki, M.; Bäumer, M.; Freund, H.-J. Size and Support Effects for CO Adsorption on Gold Model Catalysts. Catal. Letters 2003, 86, 211-219.

(27) Hammer, B.; Morikawa, Y.; Nørskov, J. K. CO Chemisorption at Metal Surfaces and Overlayers. Phys. Rev. Lett. 1996, 76, 21412144.

(28) Medford, A. J.; Vojvodic, A.; Hummelshøj, J. S.; Voss, J.; AbildPedersen, F.; Studt, F.; Bligaard, T.; Nilsson, A.; Nørskov, J. K. From the Sabatier Principle to a Predictive Theory of Transition-Metal Heterogeneous Catalysis. J. Catal. 2015, 328, 36-42.

(29) Hansen, H. A.; Shi, C.; Lausche, A. C.; Peterson, A. A.; Nørskov, J. K. Bifunctional Alloys for the Electroreduction of $\mathrm{CO} 2$ and CO. Phys. Chem. Chem. Phys. 2016, 18, 9194-9201.

(30) Herranz, T.; Deng, X.; Cabot, A.; Guo, J.; Salmeron, M. Influence of the Cobalt Particle Size in the CO Hydrogenation Reaction Studied by in Situ X-Ray Absorption Spectroscopy. J. Phys. Chem. B 2009, 113, 10721-10727.

(31) Carballo, J. M. G.; Yang, J.; Holmen, A.; García-Rodríguez, S.; Rojas, S.; Ojeda, M.; Fierro, J. L. G. Catalytic Effects of Ruthenium Particle Size on the Fischer-Tropsch Synthesis. J. Catal. 2011, 284, 102108.

(32) Wang, J. X.; Inada, H.; Wu, L.; Zhu, Y.; Choi, Y.; Liu, P.; Zhou, W.-P.; Adzic, R. R. Oxygen Reduction on Well-Defined Core-Shell Nanocatalysts: Particle Size, Facet, and Pt Shell Thickness Effects. J. Am. Chem. Soc. 2009, 131, 17298-17302.

(33) Lacroix, L.-M.; Huls, N. F.; Ho, D.; Sun, X.; Cheng, K.; Sun, S. Stable Single-Crystalline Body Centered Cubic Fe Nanoparticles. Nano Lett. 2011, 11, 1641-1645.

(34) Bonifacio, C. S.; Carenco, S.; Wu, C. H.; House, S. D.; Bluhm, H.; Yang, J. C. Thermal Stability of Core-Shell Nanoparticles: A Combined in Situ Study by XPS and TEM. Chem. Mater. 2015, 27, 6960-6968.

(35) Pitha, J. J.; Smith, A. L.; Ward, R. The Preparation of Lanthanum Oxysulfide and Its Properties as a Base Material for Phosphors Stimulated by Infrared 1. J. Am. Chem. Soc. 1947, 69, 1870-1871.
Flahaut, J.; Guittard, M.; Patrie, M. Les Oxysulfures Me2O2S Des Éléments Du Groupe Des Terres Rares. Bull. Soc. Chim. Fr. 1958, 7, 990-994.

Li, Y.; Huang, Y.; Bai, T.; Li, L. Straightforward Conversion Route to Nanocrystalline Monothiooxides of Rare Earths through a HighTemperature Colloid Technique. Inorg. Chem. 2000, 39, 34183420.

Yang, L.; Cai, Z.; Hao, L.; Xing, Z.; Dai, Y.; Xu, X.; Pan, S.; Duan, Y.; Zou, J. Nano Ce 2 O 2 S with Highly Enriched Oxygen-Deficient Ce $3+$ Sites Supported by $\mathrm{N}$ and S Dual-Doped Carbon as an Active Oxygen-Supply Catalyst for the Oxygen Reduction Reaction. ACS Appl. Mater. Interfaces 2017, 9, 22518-22529.

Tan, S.; Paglieri, S. N.; Li, D. Nano-Scale Sulfur-Tolerant Lanthanide Oxysulfide/oxysulfate Catalysts for Water-gas-Shift Reaction in a Novel Reactor Configuration. Catal. Commun. 2016, 73, 16-21.

Larquet, C.; Nguyen, A.-M.; Ávila-Gutiérrez, M.; Tinat, L.; LassalleKaiser, B.; Gallet, J.-J.; Bournel, F.; Gauzzi, A.; Sanchez, C.; Carenco, S. Synthesis of Ce $202 \mathrm{~S}$ and Gd 2(1-Y) Ce 2 Y O $2 \mathrm{~S}$ Nanoparticles and Reactivity from in Situ X-Ray Absorption Spectroscopy and X-Ray Photoelectron Spectroscopy. Inorg. Chem. 2017, 56, 14227-14236.

Carenco, S. Carbon Monoxide-Induced Dynamic Metal-Surface Nanostructuring. Chem. Eur. J. 2014, 20, 10616-10625. Grass, M. E.; Zhang, Y.; Butcher, D. R.; Park, J. Y.; Li, Y.; Bluhm, H.; Bratlie, K. M.; Zhang, T.; Somorjai, G. a. A Reactive Oxide Overlayer on Rhodium Nanoparticles during CO Oxidation and Its Size Dependence Studied by in Situ Ambient-Pressure X-Ray Photoelectron Spectroscopy. Angew. Chem. Int. Ed. Engl. 2008, 47, 8893-8896.

Tao, F.; Grass, M. E.; Zhang, Y.; Butcher, D. R.; Renzas, J. R.; Liu, Z.; Chung, J. Y.; Mun, B. S.; Salmeron, M.; Somorjai, G. a. ReactionDriven Restructuring of Rh-Pd and Pt-Pd Core-Shell Nanoparticles. Science 2008, 322, 932-934.

Grass, M. E.; Park, M.; Aksoy, F.; Zhang, Y.; Kunz, M.; Liu, Z.; Mun, B. S. Effect of O2, CO, and NO on Surface Segregation in a Rh0.5Pd0.5 Bulk Crystal and Comparison to Rh0.5Pd0.5 Nanoparticles. Langmuir 2010, 26, 16362-16367.

Papaefthimiou, V.; Dintzer, T.; Dupuis, V.; Tamion, A.; Tournus, F.; Teschner, D.; Hävecker, M.; Knop-Gericke, A.; Schlögl, R.; Zafeiratos, S. When a Metastable Oxide Stabilizes at the Nanoscale: Wurtzite CoO Formation upon Dealloying of PtCo Nanoparticles. J. Phys. Chem. Lett. 2011, 2, 900-904. Carenco, S.; Tuxen, A.; Chintapalli, M.; Pach, E.; Escudero, C.; Ewers, T. D.; Jiang, P.; Borondics, F.; Thornton, G.; Alivisatos, A. P.; et al. Dealloying of Cobalt from CuCo Nanoparticles under Syngas Exposure. J. Phys. Chem. C 2013, 117, 6259-6266.

Carenco, S.; Wu, C.-H.; Shavorskiy, A.; Alayoglu, S.; Somorjai, G. A.; Bluhm, H.; Salmeron, M. Synthesis and Structural Evolution of Nickel-Cobalt Nanoparticles Under H 2 and CO 2. Small 2015, 11, 3045-3053.

Behrens, M.; Studt, F.; Kasatkin, I.; Kühl, S.; Hävecker, M.; AbildPedersen, F.; Zander, S.; Girgsdies, F.; Kurr, P.; Kniep, B.-L.; et al. The Active Site of Methanol Synthesis over Cu/ZnO/Al2O3 Industrial Catalysts. Science 2012, 336, 893-897.

Papaefthimiou, V.; Dintzer, T.; Lebedeva, M.; Teschner, D.; Hävecker, M.; Knop-Gericke, A.; Schlögl, R.; Pierron-Bohnes, V.; Savinova, E.; Zafeiratos, S. Probing Metal-Support Interaction in Reactive Environments: An in Situ Study of PtCo Bimetallic 
Nanoparticles Supported on TiO 2. J. Phys. Chem. C 2012, 116, 14342-14349.

(50) Salmeron, M.; Schlogl, R. Ambient Pressure Photoelectron Spectroscopy: A New Tool for Surface Science and Nanotechnology. Surf. Sci. Rep. 2008, 63, 169-199.

(51) Knop-Gericke, A.; Kleimenov, E.; Hävecker, M.; Blume, R.; Teschner, D.; Zafeiratos, S.; Schlögl, R.; Bukhtiyarov, V. I.; Kaichev, V. V.; Prosvirin, I. P.; et al. X-Ray Photoelectron Spectroscopy for Investigation of Heterogeneous Catalytic Processes. Adv. Catal. 2009, 52, 213-272.

(52) Carenco, S.; Moldovan, S.; Roiban, L.; Florea, I.; Portehault, D.; Vallé, K.; Belleville, P.; Boissière, C.; Rozes, L.; Mézailles, N.; et al. The Core Contribution of Transmission Electron Microscopy to Functional Nanomaterials Engineering. Nanoscale 2016, 8, 12601279.

(53) Ersen, O.; Florea, I.; Hirlimann, C. Exploring the Nanoworld with 3D Electron Microscopy. Mater. Today 2015.

(54) Cushing, B. L.; Kolesnichenko, V. L.; O’Connor, C. J. Recent Advances in the Liquid-Phase Syntheses of Inorganic Nanoparticles. Chem. Rev. 2004, 104, 3893-3946.

(55) Diodati, S.; Dolcet, P.; Casarin, M.; Gross, S. Pursuing the Crystallization of Mono- and Polymetallic Nanosized Crystalline Inorganic Compounds by Low-Temperature Wet-Chemistry and Colloidal Routes. Chem. Rev. 2015, 115, 11449-11502.

(56) Carenco, S. Designing Nanoparticles and Nanoalloys with Controlled Surface and Reactivity. Chem. Rec. 2018.

(57) Carenco, S.; Liu, Z.; Salmeron, M. The Birth of Nickel Phosphide Catalysts: Monitoring Phosphorus Insertion into Nickel. ChemCatChem 2017, 9, 2318-2323.

(58) Carenco, S.; Surcin, C.; Morcrette, M.; Larcher, D.; Mézailles, N.; Boissière, C.; Sanchez, C. Improving the Li-Electrochemical Properties of Monodisperse Ni 2 P Nanoparticles by SelfGenerated Carbon Coating. Chem. Mater. 2012, 24, 688-697.

(59) Schaefer, Z. L.; Weeber, K. M.; Misra, R.; Schiffer, P.; Schaak, R. E. Bridging Hcp-Ni and Ni 3 C via a Ni 3 C 1-X Solid Solution: Tunable Composition and Magnetism in Colloidal Nickel Carbide Nanoparticles. Chem. Mater. 2011, 23, 2475-2480.

(60) Henkes, A. E.; Schaak, R. E. Trioctylphosphine: A General Phosphorus Source for the Low-Temperature Conversion of Metals into Metal Phosphides. Chem. Mater. 2007, 19, 42344242.

(61) Xu, L.; Liang, H.-W.; Yang, Y.; Yu, S.-H. Stability and Reactivity: Positive and Negative Aspects for Nanoparticle Processing. Chem. Rev. 2018, 118, 3209-3250.

(62) Carenco, S.; Sassoye, C.; Faustini, M.; Eloy, P.; Debecker, D. P.; Bluhm, H.; Salmeron, M. The Active State of Supported Ruthenium Oxide Nanoparticles during Carbon Dioxide Methanation. J. Phys. Chem. C 2016, 120, 15354-15361.

(63) Martínez-Prieto, L. M.; Carenco, S.; Wu, C. H.; Bonnefille, E.; Axnanda, S.; Liu, Z.; Fazzini, P. F.; Philippot, K.; Salmeron, M.; Chaudret, B. Organometallic Ruthenium Nanoparticles as Model Catalysts for CO Hydrogenation: A Nuclear Magnetic Resonance and Ambient-Pressure X-Ray Photoelectron Spectroscopy Study. ACS Catal. 2014, 4, 3160-3168.

(64) Barbaro, P.; Dal Santo, V.; Liguori, F. Emerging Strategies in Sustainable Fine-Chemical Synthesis: Asymmetric Catalysis by Metal Nanoparticles. Dalton Trans. 2010, 39, 8391-8402.

(65) Chen, T.; Rodionov, V. O. Controllable Catalysis with
Nanoparticles: Bimetallic Alloy Systems and Surface Adsorbates. ACS Catal. 2016, 6, 4025-4033.

(66) Delgado, J. A.; Benkirane, O.; Claver, C.; Curulla-Ferré, D.; Godard, C. Advances in the Preparation of Highly Selective Nanocatalysts for the Semi-Hydrogenation of Alkynes Using Colloidal Approaches. Dalt. Trans. 2017, 46, 12381-12403.

(67) Oliver-Meseguer, J.; Cabrero-Antonino, J. R.; Dominguez, I.; LeyvaPerez, A.; Corma, A. Small Gold Clusters Formed in Solution Give Reaction Turnover Numbers of 107 at Room Temperature. Science (80-. ). 2012, 338, 1452-1455.

(68) Liu, L.; Matsushita, T.; Concepción, P.; Leyva-Pérez, A.; Corma, A. Facile Synthesis of Surface-Clean Monodispersed CuOx Nanoparticles and Their Catalytic Properties for Oxidative Coupling of Alkynes. ACS Catal. 2016, 6, 2211-2221.

(69) Ressnig, D.; Moldovan, S.; Ersen, O.; Beaunier, P.; Portehault, D.; Sanchez, C.; Carenco, S. An Expeditious Synthesis of Early Transition Metal Carbide Nanoparticles on Graphitic Carbons. Chem. Commun. 2016, 52, 9546-9549.

(70) Fedorov, A.; Liu, H. J.; Lo, H. K.; Copéret, C. Silica-Supported Cu Nanoparticle Catalysts for Alkyne Semihydrogenation: Effect of Ligands on Rates and Selectivity. J. Am. Chem. Soc. 2016, 138, 16502-16507.

(71) Fiorio, J. L.; López, N.; Rossi, L. M. Gold-Ligand-Catalyzed Selective Hydrogenation of Alkynes into Cis -Alkenes via $\mathrm{H}_{2}$ Heterolytic Activation by Frustrated Lewis Pairs. ACS Catal. 2017, 7, 29732980.

(72) Ulissi, Z. W.; Singh, A. R.; Tsai, C.; Nørskov, J. K. Automated Discovery and Construction of Surface Phase Diagrams Using Machine Learning. J. Phys. Chem. Lett. 2016, 7, 3931-3935. 